\section{Radioprotective potential of histamine on rat small intestine and uterus}

\author{
E. Carabajal, ${ }^{1}$ N. Massari, ${ }^{1}$ M. Croci, ${ }^{2}$ \\ D. Martinel Lamas, ${ }^{1}$ J.P. Prestifilippo, ${ }^{3}$ \\ P. Ciraolo, ${ }^{1}$ R.M. Bergoc, ${ }^{1,2}$ E.S. Rivera, ${ }^{1}$ \\ V.A. Medina ${ }^{1,4}$ \\ 'Laboratory of Radioisotopes, School of \\ Pharmacy and Biochemistry, University \\ of Buenos Aires; \\ IInstitute of Immunooncology, Buenos \\ Aires; \\ ${ }^{3}$ Physiopathology Department, School \\ of Pharmacy and Biochemistry, \\ University of Buenos Aires; \\ ${ }^{4}$ National Scientific and Technical \\ Research Council (CONICET), Buenos \\ Aires, Argentina
}

\section{Abstract}

The aim of this study was to improve knowledge about histamine radioprotective potential investigating its effect on reducing ionising radiation-induced injury and genotoxic damage on the rat small intestine and uterus. Forty 10-week-old male and 40 female SpragueDawley rats were divided into 4 groups Histamine and histamine-5Gy groups received a daily subcutaneous histamine injection (0.1 $\mathrm{mg} / \mathrm{kg}$ ) starting $24 \mathrm{~h}$ before irradiation. Histamine-5Gy and untreated-5Gy groups were irradiated with a dose of whole-body Cesium137 irradiation. Three days after irradiation animals were sacrificed and tissues were removed, fixed, and stained with haematoxylin and eosin, and histological characteristics were evaluated. Proliferation, apoptosis and oxidative DNA markers were studied by immunohistochemistry, while micronucleus assay was performed to evaluate chromosomal damage. Histamine treatment reduced radiation-induced mucosal atrophy, oedema and vascular damage produced by ionising radiation, increasing the number of crypts per circumference $(239 \pm 12$ vs $160 \pm 10 ; \mathrm{P}<0.01)$. This effect was associated with a reduction of radiation-induced intestinal crypts apoptosis. Additionally, histamine decreased the frequency of micronuclei formation and also significantly attenuated $8-0 \mathrm{HdG}$ immunoreactivity, a marker of DNA oxidative damage. Furthermore, radiation induced flattening of the endometrial surface, depletion of deep glands and reduced mitosis, effects that were completely blocked by histamine treatment. The expression of a proliferation marker in uterine luminal and glandular cells was markedly stim- ulated in histamine treated and irradiated rats.

The obtained evidences indicate that histamine is a potential candidate as a safe radioprotective agent that might increase the therapeutic index of radiotherapy for intra-abdominal and pelvic cancers. However, its efficacy needs to be carefully investigated in prospective clinical trials.

\section{Introduction}

Radiotherapy is today considered as one of the most important weapon in the fight against cancer, alone or associated to other treatments and relies on ionising radiationinduced DNA damage to kill malignant cells. About eighty percent of cancer patients need radiotherapy at some time or other, either for curative or palliative purposes. To optimise results, it is required a cautious balance between the total dose of radiotherapy delivered and the threshold limit of the surrounding normal critical tissues. In order to obtain better tumour control with a higher dose, the normal tissues should be protected against radiation damage. Therefore, radiobiologists have long been interested in identifying novel, nontoxic, effective, and convenient compounds to protect humans against radiation-induced normal tissue injuries. ${ }^{1-4}$

A large number of drugs have been screened for radioprotective efficacy, over the past 50 years. However, no suitable drug has yet been introduced for routine clinical use. ${ }^{1,5}$

Potentially serious complications are an inevitable consequence of radical therapies. Patients who undergo pelvic or abdominal radiotherapy may develop acute and/or chronic side effects, resulting from gastrointestinal tract, and bone marrow alterations., $1,6,7$ Radiotherapy initially causes mucosal changes characterised by inflammation or cell death, but subsequently persistent cytokine activation in the submucosa leads to progressive ischaemia, fibrosis and loss of stem cells. ${ }^{6}$ The acute depletion of bone marrow components following irradiation have been attributed to the direct effect of radiation depleting the stem cell compartment, whereas the chronic bone marrow aplasia following initial repopulation has been ascribed to stromal damage thought secondary to an impaired vascular supply and subsequent fibrosis, with destruction of fine vasculature followed by fatty marrow replacement of the normal hematopoietic marrow. ${ }^{1,7}$

Furthermore, female patients may display uterine damage, including endometrial and myometrial atrophy, scar fibrosis and hypovascularization, which are negative factors for the establishment and maintenance of a pregnancy and for a convenient labour., ${ }^{8,9}$
Correspondence: Dr. Vanina A. Medina Laboratory of Radioisotopes, School of Pharmacy and Biochemistry, University of Buenos Aires, Junín 956, Buenos Aires, Argentina 1113.

Tel. $+54.11 .49648277 / 8202$

Fax: +54.11.49648277/8202 ext: 31 .

E-mail: vmedina@ffyb.uba.ar.

Key words: histamine, ionising radiation, radioprotectors, small intestine, uterus.

Contributions: VAM, research design; EC, NM, MC, DML, DL, JPP, RMB, ESR, VAM, research performing; VAM, ESR, participated in acquisition of funding; EC, NM, MC, VAM, data analysis; ESR, JPP, VAM, involved in manuscript editing and critical review; VAM, EC, NM, manuscript writing.

Acknowledgements: this work has been supported by grants from the University of Buenos Aires, 20020090300039, 20020100100270, Fundación Alberto J. Roemmers, the National Agency of Scientific and Technological Promotion BID PICT-2007-01022, CONICET (PIP 112201101 01121), and from the EU-FP7 COST Action BM0806 (Recent advances in histamine receptor H4R research).

The authors thank the National Cancer Institute of Argentina for financial support through a fellowship to Eliana Carabajal. The technical assistance of Alejandro Paredes is appreciated. The authors dedicate this work to the memory of their friend and colleague, Dr. Maximo Croci.

Received for publication: 14 May 2012. Accepted for publication: 8 September 2012.

This work is licensed under a Creative Commons Attribution NonCommercial 3.0 License (CC BYNC 3.0).

(OCopyright E. Carabajal et al., 2012

Licensee PAGEPress, Italy

European Journal of Histochemistry 2012; 56:e48 doi:10.4081/ejh.2012.e48

In this regard, it has been previously reported that histamine significantly protects two of the most radiosensitive tissues, mouse small intestine and mouse and rat bone marrow, from high doses of gamma radiation. ${ }^{10,11}$ In addition, histamine prevents morphological and functional radiation-induced damage on rat submandibular gland. ${ }^{12}$

Histamine (2-(imidazol-4-yl) ethylamine) is a biogenic amine widely distributed throughout the body with a broad spectrum of activities in numerous physiological and pathological situations, and is synthesised by the histidine decarboxylase enzyme (HDC)..$^{13}$ The expression pattern of histamine receptors in the intestinal tract in which histamine regulates multiple functions, including contraction of intestinal smooth muscle and gastric acid secretion was previously demonstrated..$^{14,15}$ Furthermore, it is suggested that histamine is 
important for normal ovulation, blastocyst implantation, placental blood flow regulation, lactation and contractile activity of uterus. ${ }^{16}$ In the murine uterus the rapidly dividing epithelial cells of the endometrium can be defined as the major sources of histamine. In these cells, the level of HDC expression is controlled mainly by progesterone-mediated signals which, interestingly, induce maximal level of HDC expression on the day of implantation (day four of pregnancy)..$^{17}$

The biological effects of ionising radiation results principally from damage to DNA and double-strand breaks, which can lead to chromosomal aberrations, are the most relevant lesions responsible for most biologic insults, including cell killing. ${ }^{1}$ Micronuclei in interphase mammalian cells are reliable biomarkers for evaluating ionising radiation-induced chromosome damage. ${ }^{18}$ In addition, ionising radiation through the indirect action, initiates intracellular oxidative stress through enhanced formation of reactive oxygen species that assault DNA. 8-Hydroxy-2'-deoxyguanosine $(8-0 \mathrm{HdG})$ is a major type of oxidative DNA damage, and is often analysed as a marker of oxidative stress in human and animal studies of genotoxic activity. ${ }^{19}$

Based on our previous data on the histamine radioprotective effect on mouse small intestine, bone marrow and salivary glands; in the present work we aimed to improve our knowledge about histamine radioprotective potential, investigating its effect on reducing the ionising radiation-induced injury and genotoxic damage on the rat small intestine and uterus. For that purpose, we evaluated the histological characteristics and markers of proliferation, apoptosis, and DNA damage in whole body irradiated-rats.

\section{Materials and Methods}

\section{Treatment and irradiation}

Forty 10-week-old male and 40 female Sprague-Dawley rats, weighing 200-230 g were purchased from the Division of Laboratory Animal Production, School of Veterinary Sciences, University of La Plata, Buenos Aires and were randomly separated into 4 groups per gender ( $\mathrm{n}=10$ each). Rats were maintained in our animal health care facility at 22 to $24^{\circ} \mathrm{C}$ and $50 \%$ to $60 \%$ humidity on a 12 hours light/dark cycle with food and water available ad libitum. To determine the radioprotective potential of histamine on rat small intestine and uterus, the same experimental procedures that we previously described in detail were used. . $^{10-12}$

Histamine and histamine-5 Gray (Gy) groups received a daily subcutaneous histamine injection $(0.1 \mathrm{mg} / \mathrm{kg})$ starting $24 \mathrm{~h}$ before irradiation and which continued until the end of the experimental period (3 days post irradiation) while untreated groups received saline. Histamine-5 Gy and untreated-5 Gy groups were irradiated using Cesium-137 source (IBL 437C type $\mathrm{H}$ ) of $189 \mathrm{TBq}$ (dose rate: $7 \mathrm{~Gy} / \mathrm{min}$ ) with a single whole-body dose of $5 \mathrm{~Gy}$.

Three days post irradiation animals were sacrificed by cervical dislocation and tissues were removed, and histological and histochemical characteristics were evaluated $(n=10$ each group).

Animal procedures were in accordance with recommendations from the Guide for the Care and Use of Laboratory Animals of the National Research Council, USA, 1996, and protocols were approved by the Ethical Committee for the Use and Care of Laboratory Animals of the School of Pharmacy and Biochemistry and also by Ethical and Educational Committee of the Institute of Immunooncology.

\section{Histopathological studies}

Small intestine was opened along the mesenteric border and samples were pinned flat on corkboard, mucosal side up, to allow immediate fixation with $10 \%$ neutral buffered formalin and comparable samples. Uterus was

Table 1. Histopathological characteristics of the rat small intestine.

\begin{tabular}{lcccc} 
Group & Trophism & Crypts/circumference* & Ulceration & Other changes \\
Untreated $^{\circ}$ & Normal & $220 \pm 12$ & Absent & - \\
Histamine $^{\#}$ & Normal & $242 \pm 10$ & Absent & - \\
\hline Untreated-5 Gy & Marked atrophy & $160 \pm 10^{\mathrm{a}}$ & Severe Anisokaryosis/Oedema & \\
Histamine-5 Gy^ & Moderate atrophy & $239 \pm 12^{\mathrm{b}}$ & Moderate & -
\end{tabular}

*The mean values of the experimental group were calculated as the average of the number of crypts examined in 10 fields.

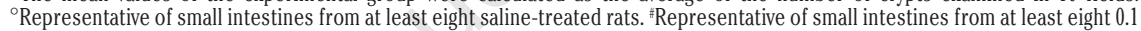
$\mathrm{mg} / \mathrm{kg}$.day histamine-treated rats; ${ }^{~}$ Representative of small intestines from at least eight saline-treated and 5 Gy-irradiated rats; $\wedge$ Representative of small intestines from at least eight histamine-treated and 5 Gy-irradiated rats. ${ }^{a} \mathrm{P}<0.01$ vs untreated, ${ }^{\mathrm{b}}<<0.01$ vs untreated-5 Gy (ANOVA and Tukey Post test).

Table 2. Evaluation of apoptosis in the small intestine.

\begin{tabular}{ll} 
Group & Apoptotic cells/crypts* \\
Untreated $^{\circ}$ & $0.3 \pm 0.1$ \\
Histamine $^{\#}$ & $0.3 \pm 0.2$ \\
\hline Untreated-5 Gy $^{\S}$ & $1.7 \pm 0.3^{\mathrm{a}}$ \\
Histamine-5 Gy^ & $0.4 \pm 0.1^{\mathrm{b}}$ \\
\hline
\end{tabular}

*The number of TUNEL positive cells per crypt expressed as mean \pm SEM of 10 fields examined. ${ }^{\circ}$ Representative of small intestines from at least eight saline-treated rats. "Representative of small intestines from at least eight 0.1 $\mathrm{mg} / \mathrm{kg}$.day histamine-treated rats; 'Representative of small intestines from at least eight saline-treated and 5 Gy-irradiated rats; ^Representative of small intestines from at least eight histamine-treated and 5 Gy-irradiated rats. $\mathrm{P}<0.001$ us untreated, ${ }^{\mathrm{b}}<0.001$ us untreated-5 Gy (ANOVA and Tukey Post test).

Table 3. Evaluation of 8-hydroxydeoxyguanosine in the small intestine.

\begin{tabular}{lc} 
Group & 8-OHdG-positive cells* \\
Untreated $^{\circ}$ & $21.9 \pm 0.8$ \\
Histamine $^{\sharp}$ & $21.4 \pm 1.5$ \\
\hline Untreated-5 Gy $^{\S}$ & $46.7 \pm 4.9^{\mathrm{a}}$ \\
Histamine-5 Gy^ $^{\wedge}$ & $7.5 \pm 0.8^{\mathrm{a}, \mathrm{b}}$ \\
\hline
\end{tabular}

*The percentage of 8-hydroxydeoxyguanosine (8-OHdG) immunoreactivity in cryptal cells expressed as mean \pm SEM o 10 fields examined. ${ }^{\circ}$ Representative of small intestines from at least eight saline-treated rats. "Representative of small intestines from at least eight $0.1 \mathrm{mg} / \mathrm{kg}$.day histamine-treated rats; sRepresentative of small intestines from at least eight salinetreated and 5 Gyirradinted treated and 5 Gyinraliated intestines from at least eight histamine-001 ated rats. ${ }^{\mathrm{a}} \mathrm{P}<0.001$ vs untreated, ${ }^{\mathrm{b}} \mathrm{P}<0.001$ vs untreated $-5 \mathrm{G}$ (ANOVA and Tukey Post test).
Table 4. Analysis of micronucleus frequency in the small intestine.

\begin{tabular}{lc} 
Group & Micronuclei* \\
Untreated $^{\circ}$ & - \\
Histamine $^{\sharp}$ & - \\
\hline Untreated-5 Gy $^{\S}$ & $50 \pm 2^{\mathrm{a}}$ \\
Histamine-5 Gy^ & $30 \pm 2^{\mathrm{a}, \mathrm{b}}$ \\
\hline
\end{tabular}

*Chromosomal damage was measured by analysing micronucleus. The number of micronuclei was determined in 1000 cells and is expressed as mean \pm SEM. "Representative of small intestines from at least eight $0.1 \mathrm{mg} / \mathrm{kg}$.day histamine-treated rats; \$Representative of small intestines from at least eight saline-treated and 5 Gy-irradiated rats; ${ }^{\wedge}$ Representative of small intestines from at least eight histamine-treated and 5 Gyirradiated rats. ${ }^{\mathrm{a}}<0.001$ vs untreated, ${ }^{\mathrm{D}} \mathrm{P}<0.001$ vs untreated-5 Gy (ANOVA and Tukey Post test).

Table 5. Evaluation of mitotic figures in the uterus.

\begin{tabular}{lc} 
Group & Number of mitosis/field* \\
Untreated $^{\circ}$ & $2.4 \pm 0.2$ \\
Histamine $^{\sharp}$ & $2.3 \pm 0.2$ \\
\hline Untreated-5 Gy $^{\S}$ & $0.4 \pm 0.1^{\mathrm{a}}$ \\
Histamine-5 $\mathrm{Gy}^{\wedge}$ & $1.7 \pm 0.1^{\mathrm{a}, \mathrm{b}}$
\end{tabular}

*The number of mitosis per x630-magnifitation field expressed as mean + SEM of 10 fields examined. ${ }^{\circ}$ Representative of small intestines from at least eight saline-treated rats. *Representative of small intestines from at least eight 0.1 "Representative of small intestines from at least eight 0.1
$\mathrm{mg} / \mathrm{kg}$.day histamine-treated rats; ${ }^{\text {\& }}$ Representative of small $\mathrm{mg} / \mathrm{kg}$.day histamine-treated rats; ${ }^{8}$ Representative of small
intestines from at least eight saline-treated and 5 Gy-irradiated rats; ^ Representative of small intestines from at least eight histamine-treated and 5 Gy-irradiated rats. ${ }^{\mathrm{a}}<<0.001$ s untreated, ${ }^{\mathrm{b}} \mathrm{P}<0.001$ vs untreated-5 Gy (ANOVA and Tukey Post test). 
removed and fixed with $10 \%$ neutral buffered formalin. Tissue samples were embedded in paraffin and cut into $4 \mu \mathrm{m}$ thick serial sections. Small intestine and uterus morphology and histopathological characteristics were examined on tissue sections after haematoxylin-eosin staining.

Parameters analyzed in the proximal part of the small intestine were: i) mucosal trophism (Normal: normal appearance of mucosal villi and crypts; Moderate atrophy: slight reduction in the number of villi and crypts only confirmable through crypt comparative counting but conserving histological normal appearance; Marked atrophy: severe reduction in the number of villi and crypts with morphological changes in shape and structure); ii) number of intestinal crypts per circumference; iii) mucosal superficial ulceration (Absent: total continuity of cells layers covering the mucosal villi; Moderate: occasional loss of continuity in the epithelial covering of villi with mild inflammatory response; Severe: significant number of mucosal ulcers with important inflammatory response and haemorrhages; iv) nuclear and cytoplasmic changes (Absent: adequate nucleus/cytoplasmic ratio; Mild anisokaryosis: slight alteration in the nucleus/cytoplasmic ratio and different nuclear size between mucosal cells; Anisokaryosis/anisocytosis: marked alteration of the nucleus/cytoplasmic ratio, important difference in the nuclear and cytoplasmic size between mucosal cells); v) villous oedema (Absent: total absence of oedematous sectors; Present: oedematous separation of the mucosal layers by clear sectors in the histological preparation); vi) vascular damage (Absent: normal appearance of the vascular structures; Present: vascular congestion, proliferation and oedema of endothelial cells, occasional obliteration and thrombosis).

\section{Immunohistochemical staining}

After deparaffinization, specimens were placed in citrate buffer $(10 \mathrm{mM}, \mathrm{pH} \mathrm{6.0)}$ and heated at boiling temperature in a microwave oven twice for 2 min for antigen retrieval. Endogenous peroxidase activity was blocked with $3 \% \mathrm{H}_{2} \mathrm{O}_{2}$ in distilled water. After blocking, tissues were incubated with primary rabbit anti histamine (1:100, Sigma Chemical Co., St. Louis, MO, USA), mouse anti proliferating cell nuclear antigen (PCNA, 1:100, Dako Cytomation, Glostrup, Denmark), and goat anti 8-0HdG (1:200, Millipore, Temecula, CA, USA) antibodies overnight in a humidified chamber at $4^{\circ} \mathrm{C}$. Immunoreactivity was detected by using horseradish peroxidase-conjugated anti-mouse, anti-goat or anti-rabbit antibodies, as appropriate, and visualized by diamino-benzidine staining (Sigma Chemical Co.). To evaluate sub-cellular localization of these proteins, nuclei were stained with haematoxylin.
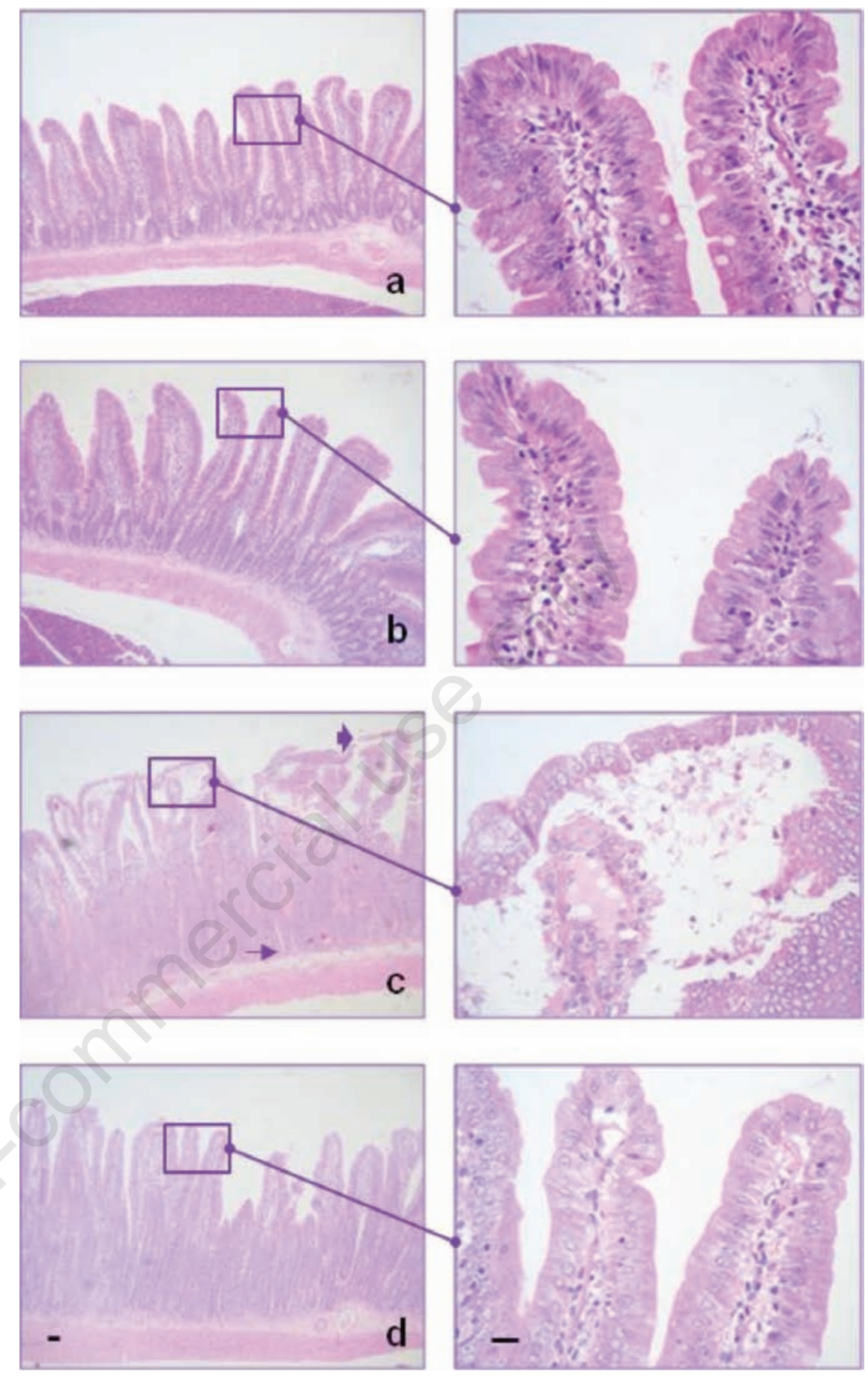

Figure 1. Histamine prevents damage produced by ionising radiation in the small intestine. Normal appearance of the small bowel in A) untreated and B) histamine treated animals. C) Intestine of irradiated rat showing reduction in the number of crypts (arrow), mucosal ulceration and villous oedema (arrowhead). D) Intestine of irradiated and histamine treated rat, exhibiting preservation of the mucosa, absence of vascular damage and an increased number of crypts. Haematoxylin-eosin. 100x and 630x magnification. Scale bar: $20 \mu \mathrm{m}$. 
Light microscopy was performed on an Axiolab Karl Zeiss microscope (Göttingen, Germany). Photographs were taken at $100 x$, 400x and 630x magnifications using a Canon PowerShot G5 camera (Tokyo, Japan). To control the signal specificity, serial sections were made from five selected positive cases that were subjected to the same staining procedure, with either a normal mouse or rabbit IgG or PBS to replace the first antibody. No signal was detected in this control staining.

\section{Determination of apoptosis}

Apoptotic cells were determined by terminal deoxynucleotidyl transferase (TdT) mediated deoxyuridine triphosphate biotin nick end labelling (TUNEL) assay. Fragmented DNA in cells undergoing apoptosis was detected using Apoptag $^{\mathrm{TM}}$ plus peroxidase in situ apoptosis Detection Kit (CHEMICON International, Temecula, CA, USA) according to the manufacturer's instructions.

Samples were visualized using an Axiolab Karl Zeiss microscope. All photographs were taken at $630 \mathrm{x}$ magnification using a Canon PowerShot G5 camera. Negative control sections were incubated in the absence of TdT. Results were expressed as the number of TUNEL-positive cells per field of at least 15 fields examined.

\section{Micronucleus assay}

Micronucleus assay was performed according to Vanhauwaert et al. ${ }^{20}$ with adaptation. Briefly, animals were sacrificed, a segment of the proximal jejunum was excised and flushed free of faeces with PBS. The small intestine was opened along the mesenteric border mucosal side up and was placed onto clean glass slides by imprint technique, and then allowed to dry. Finally, cells were stained with May-Grunwald Giemsa stain and visualised under Axiolab Karl Zeiss microscope.

At least 1000 small intestine epithelial cells, in which the nucleus was well identified but not fragmented, and surrounded by cytoplasm with a clear boundary, were analyzed per animal for micronucleus incidence. The criteria of the micronucleus were: i) the same staining as the main nucleus; ii) smaller than 1/3 diameter of the main nucleus; iii) not attached to the main nucleus. ${ }^{20,21}$

\section{Statistical analysis}

Data shown are mean \pm standard error of the mean (SEM). Statistical evaluations were made by analysis of variance (ANOVA) followed by Tukey Multiple Comparison Test. P values $<0.05$ were considered significant. All statistical analyses were performed with GraphPad Prism Version 5.00 software (San Diego, CA, USA).
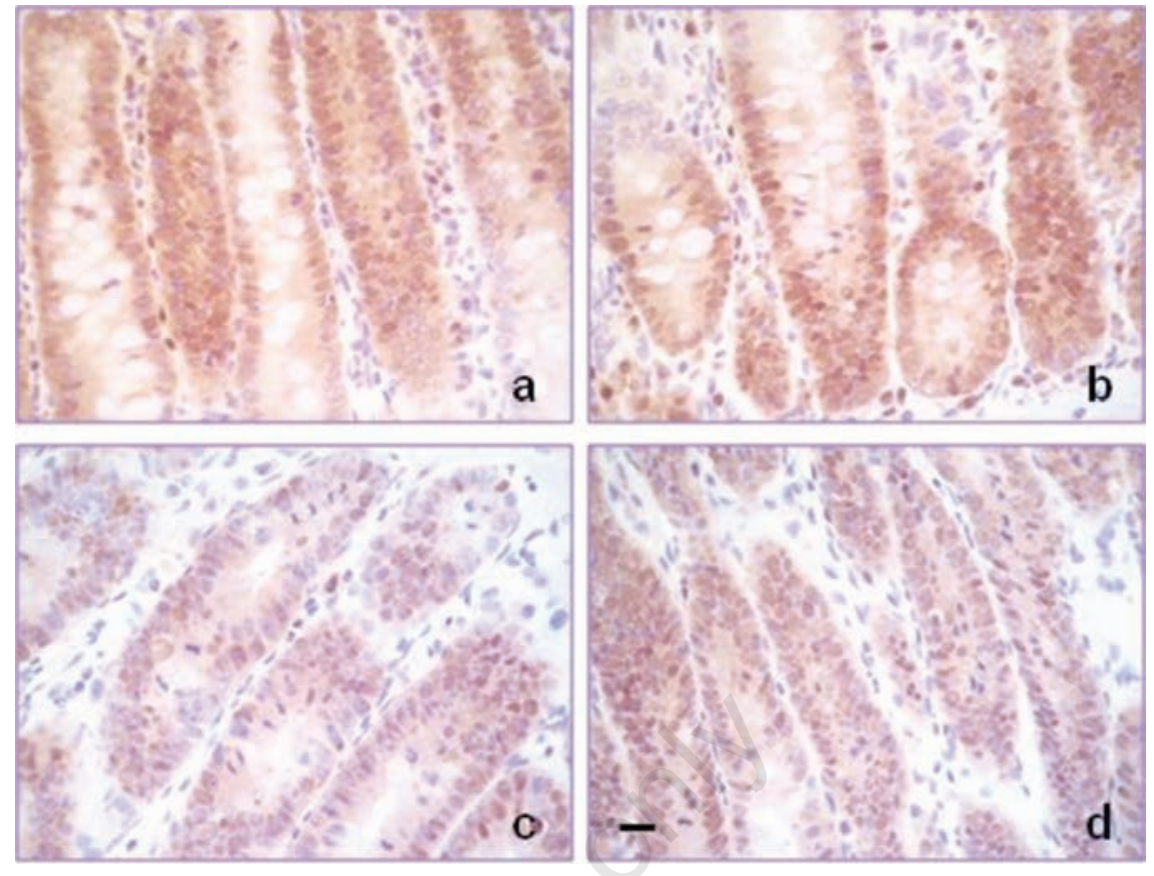

Figure 2. PCNA expression in the small intestine. High PCNA immunoreactivity in intestine of A) untreated, B) histamine treated, C) irradiated, rats D) irradiated and histamine treated. 630x magnification. Scale bar: $20 \mu \mathrm{m}$.
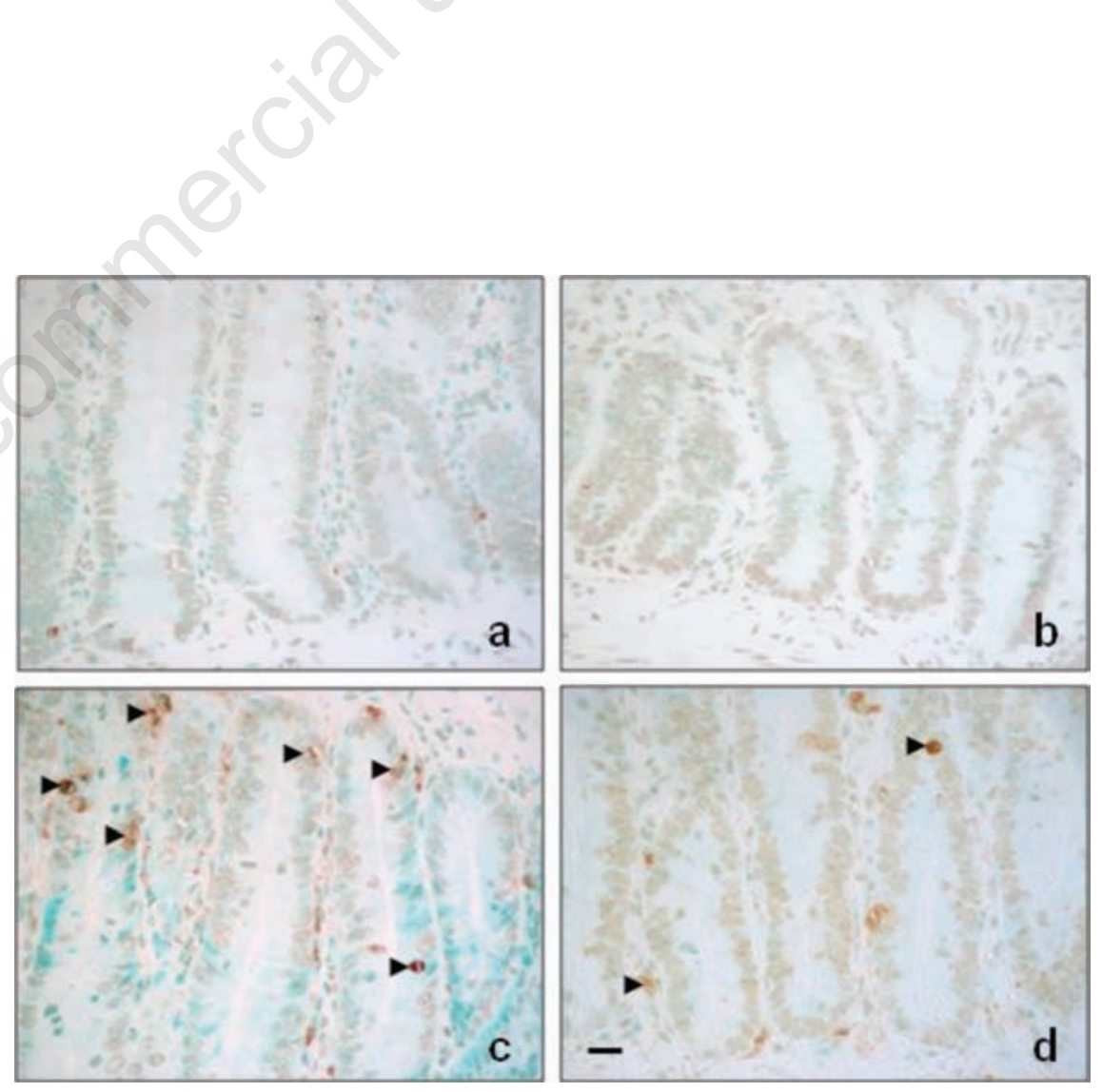

Figure 3. Histamine reduces ionising radiation-induced apoptosis of cryptal cells. Intestinal crypts of A) untreated and B) histamine treated rats showing little or no apoptosis. C) Irradiated rat intestinal crypts demonstrating a higher number of apoptotic cells (arrowhead). D) Irradiated and histamine treated rat intestinal crypts showing fewer apoptotic cells (arrowhead). 630x magnification. Scale bar: $20 \mu \mathrm{m}$. 


\section{Results}

\section{Effect of histamine on the histological characteristics of the small intestine}

One of the most widely used experimenta techniques to assess the biological responses against a dose of ionising radiation and to study potential radioprotectors, is the one developed by Withers and colleagues based on the observation of regenerating intestinal crypts in whole body irradiated animals. The score of radiation damage is the number of regenerating crypts per circumference of the sectioned intestine three days post irradiation. ${ }^{1}$ As indicated in Table 1, treatment with histamine decreased mucosal atrophy, oedema and vascular damage produced by ionising radiation. It also preserved the villi, crypts and nuclear characteristics of the small intestine after exposure to ionising radiation (Figure 1). The number of crypts per circumference in irradiated and histamine treated rats was significantly higher than in the group of irradiated and untreated animals $(239 \pm 12$ vs $160 \pm 10$, $\mathrm{P}<0.01$ ). Importantly, the number of crypts per circumference in irradiated animals but treated with histamine did not differ from the value obtained in non-irradiated animals (Table 1). In addition, histological features of small intestine were not altered by histamine treatment in non-irradiated animals (Table 1 ; Figure 1).

\section{Effect of histamine on the intestinal cell proliferation and apoptosis}

Histamine treatment significantly preserved the histological characteristics of intestinal crypts, which are structures containing pluripotent stem cells that allow continuous supply of all epithelial cells of the small intestine. In order to determine whether histamine effect was related to the modulation of proliferation and apoptosis, these biological processes were investigated through the determination of PCNA by immunohistochemistry and apoptosis by the TUNEL assay.

Results indicate that PCNA expression was high and restricted to the crypts in the small intestine of untreated rats and no significant differences were observed after histamine treatment (Figure 2). By contrast, the number of apoptotic cells in the crypts was low and treatment with histamine in non-irradiated animals did not modify the number of apoptotic cells (Figure 3; Table 2). PCNA expression was also elevated in the irradiated group of animals and histamine did not produce significant changes (Figure 2). On the other hand, ionising radiation increased the number of apoptotic crypt cells while treatment with histamine significantly reduced it $(0.4 \pm 0.1 \mathrm{vs}$ $1.5 \pm 0.3, \mathrm{P}<0.001)$, reaching values that were comparable with the ones of non-irradiated animals (Figure 3; Table 2).

\section{Effect of histamine on the intestinal genotoxic damage}

Whole body exposure of rats to gamma-radiation resulted in damage to cellular DNA, evidenced by an enhanced oxidative DNA damage and also induced the formation of micronuclei in small intestine (Figure 4; Tables 3 and 4). Administration of histamine prior to the radiation exposure prevented the radiation induced DNA oxidative damage, reducing the percentage of 8 - $0 \mathrm{HdG}$ positivity in cryptal cells $(7.5 \pm 0.8$ vs $46.7 \pm 4.9, \mathrm{P}<0.001$ ) (Figure 4 ; Table 3). Furthermore, histamine treatment reduced the frequency of micronuclei in small intestine in whole body irradiated animals (Table 4).

\section{Effect of histamine on the intracel- lular histamine immunoreactivity of the small intestine}

Figure 5 shows the results of the immunohistochemical analysis of intracellular histamine. Histamine content remained unchanged in the crypts of histamine treated and also of
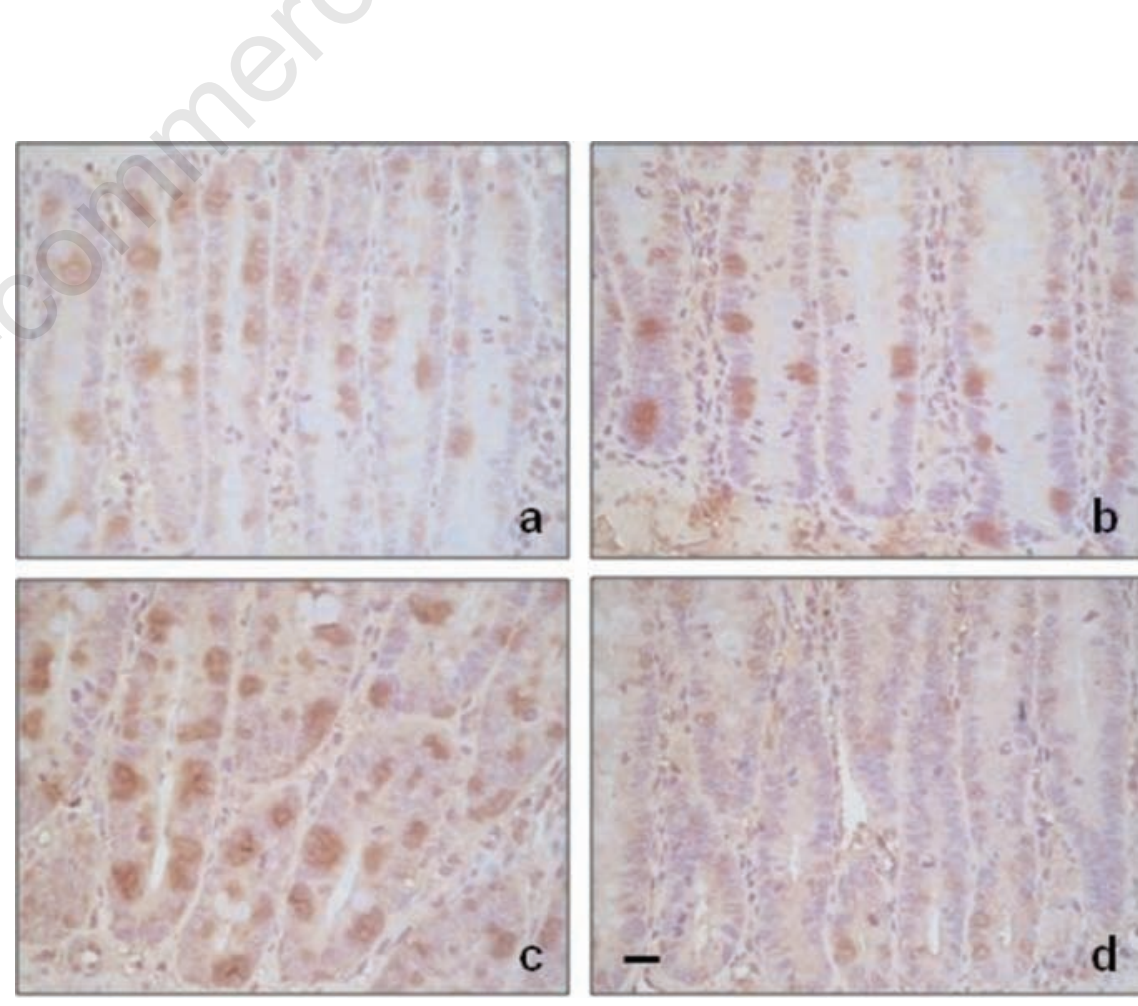

Figure 4. Histamine reduces ionising radiation-induced 8-OHdG labelling in cryptal cells. Intestinal crypts of A) untreated and B) histamine treated rats showing modest 8-OHdG immunoreactivity. C) Irradiated rat intestinal crypts demonstrating a higher number of 8 OHdG positive cells. D) Irradiated and histamine treated rat intestinal crypts showing fewer 8-OHdG positive cells. 630x magnification. Scale bar: $20 \mu \mathrm{m}$. 
ation. Conversely, histamine treatment in irradiated rats markedly stimulated the expression of PCNA in both cell types (Figure 7).

\section{Effect of histamine on the intracel- lular histamine immunoreactivity of the uterus}

Uterus of non-irradiated rats demonstrated moderate histamine intracellular levels and the ionising radiation markedly increased its histamine content (Figure 8).

\section{Discussion}

The tolerance of normal tissues for radiation depends on the ability of clonogenic cells to maintain a sufficient number of mature cells to preserve the function of the organ. Casarett has suggested a classification of mammalian cell radiosensitivity based on histological observation of early cell death and has divided cells into four categories. Group I of Casarett's classification, the most sensitive group, includes intestinal crypt cells and bone marrow stem cells. ${ }^{1}$

Some of the signs of gastrointestinal syndrome evidenced after whole-body irradiation with doses of 5-10 Gy include anorexia, lethargy, diarrhoea, decreased body fluids and electrolytes, weight loss, reduced white blood cells, bleeding and bacteraemia, aggravating the injury and contributing to the death. Severe enteritis occurs about 2-5 days post-irradiation. ${ }^{1,6}$ In order to contribute to better understand the role of histamine in the ionising radiation-induced biological effects on small intestine, we investigated its effects on other mammalian species.

The results clearly demonstrate that histamine treatment significantly prevents the ionising radiation-induced toxicity on small intestine, decreasing the histological damage and improving the trophism of the enterocytes. Histamine completely avoids the decrease in the number of crypts promoted by ionising radiation, effect that is essential to achieve intestinal mucosa recovery. ${ }^{1,22,23}$

In rapidly proliferating tissues, such as the lining of the small intestine, there is a tight regulation of cell growth and death by apoptosis, which is fundamental to the maintenance of tissue homeostasis. ${ }^{22,24}$ The radiation damage to stem cells has serious repercussions because they were programmed to divide many times to maintain a large population, and if they lose their potential descendants are lost from the population. ${ }^{1,22,23}$ In this regard, we investigated whether histamine could modulate the induction of apoptosis caused by ionising radiation. Histamine significantly reduces the ionising radiation-induced apoptosis in crypt cells. Coincidentally, we have previously reported that histamine prevents ionising radiation-induced injury in mouse small intestine by increasing proliferation of damaged intestinal mucosa and inhibiting apoptosis that was associated with an augmented antioxidant enzyme levels and histamine content ${ }^{10} \mathrm{In}$ this line, previous studies showed that increased levels of histamine produced by the treatment with aminoguanidine, a diamine oxidase inhibitor, reduces ischemia-induced intestinal mucosa cell apoptosis. This result might be partially supported by the fact that histamine, working as a growth factor, accelerated repair of damaged mucosa in the rat small intestine..$^{25,26}$

In addition, we evaluated the expression of
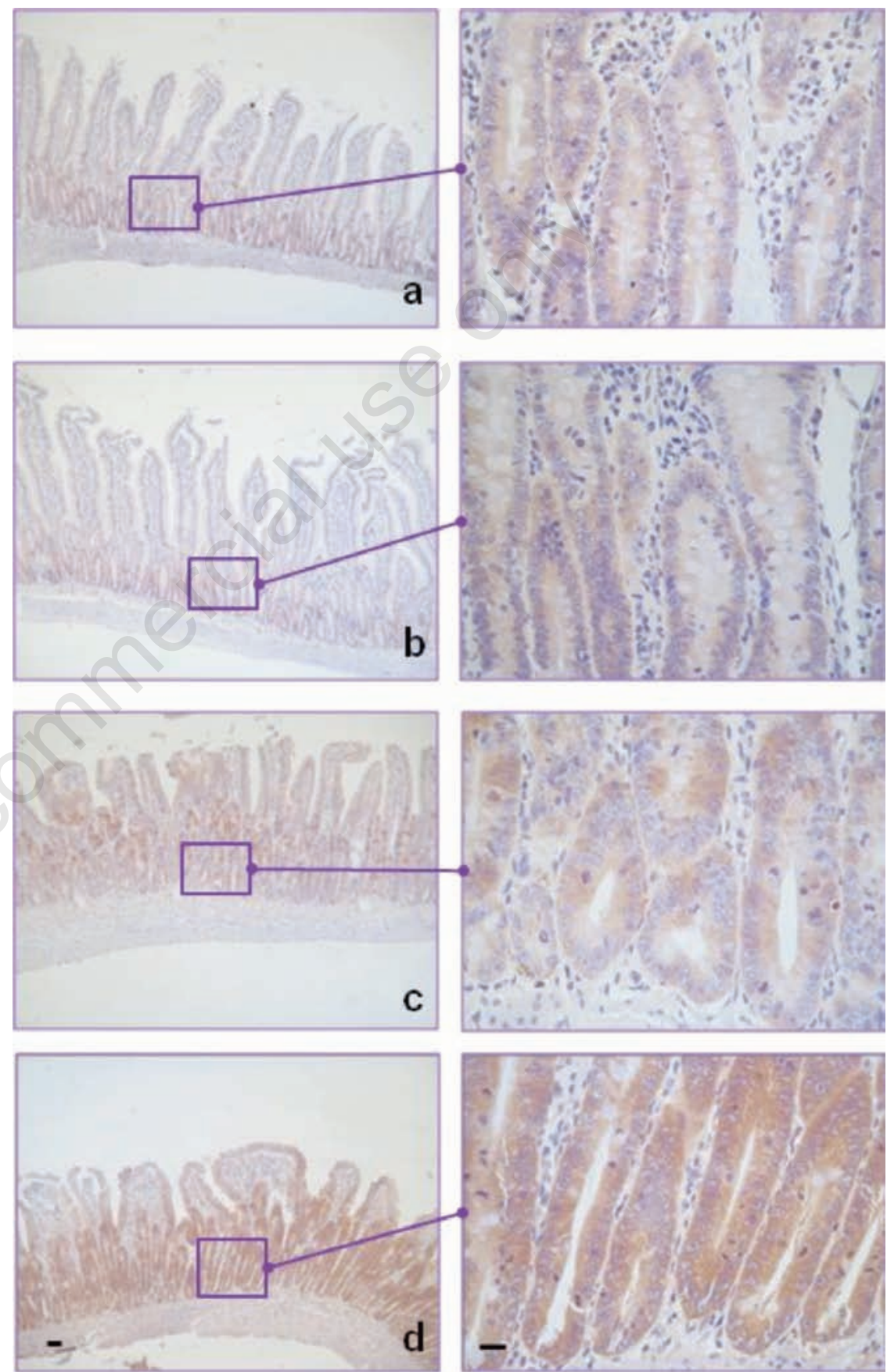

Figure 5. Histamine enhances intestinal histamine content in irradiated animals. Moderate intracellular content of histamine in rat intestinal crypts of A) untreated, B) histamine treated, and C) irradiated rats. D) Elevated intracellular histamine content in crypts of irradiated and histamine treated rats. 100x and 630x magnification. Scale bar: $20 \mu \mathrm{m}$. 
PCNA as a proliferation marker. Results showed that the PCNA immunoreactivity is not significantly modified by histamine treatment neither in irradiated nor in non-irradiated intestinal cells, indicating that the intestinal crypts are regenerating after 3 days post-irradiation, which suggests that the radioprotective effect of histamine in rat small intestine is mainly associated with the inhibition of apoptosis. In agreement with this, we recently reported that histamine markedly prevented radiation injury on submandibular gland, ameliorating the histological and functional alterations. Histamine prevented radiation-induced toxicity in submandibular gland essentially by suppressing apoptosis of ductal and acinar cells, reducing the number of apoptotic cells per field. ${ }^{12}$ Exposure to ionising radiation induced chromosome damage and oxidative stress, which can lead to cell death.,18,19

Therefore, we further investigated genotoxic activities by analysing micronucleus frequency and $8-0 \mathrm{HdG}$ immunoreactivity in small intestinal cells. Results show that histamine has protective action against radiationinduced oxidative DNA damage in vivo, reducing the frequency micronuclei formation and also significantly attenuated $8-0 \mathrm{HdG}$ immunoreactivity, a marker of DNA oxidative damage.

We also evaluated the content of histamine in the intestinal cells. The results indicate that histamine treatment increases the intracellular content of histamine especially in the intestinal crypts of irradiated rats as we have previously observed in mouse small intestine. ${ }^{10}$ These results suggest that endogenous histamine might also be acting as a free-radical scavenger, as it was previously described for other compounds with similar chemical structure that includes an imidazol group. ${ }^{27}$

Ionising radiation also affects other organs in the pelvic region and abdomen such as the uterus, which is considered as group II of the classification of tissue radiosensitivity of Casarett. ${ }^{1}$ Uterine damage that includes atrophy of the endometrial glands and stromal, impaired growth and blood flow, is a likely consequence of pelvic irradiation. Therefore, women treated with radiation have an increased rate of uterine dysfunction leading to miscarriage, mid-trimester pregnancy loss, preterm labour, low birth weight, and placental abnormalities. ${ }^{1,9,28}$ Therefore, we investigated the effect of histamine on irradiated uterus. Histopathological analysis demonstrated that histamine treatment prevents the ionising radiation-induced flattening of the endometrial surface together with the reduction of deep glands and mitosis. This effect was associated with an increased PCNA immunoreactivity in the luminal and glandular endometrial cells. In agreement with this result, we have previously
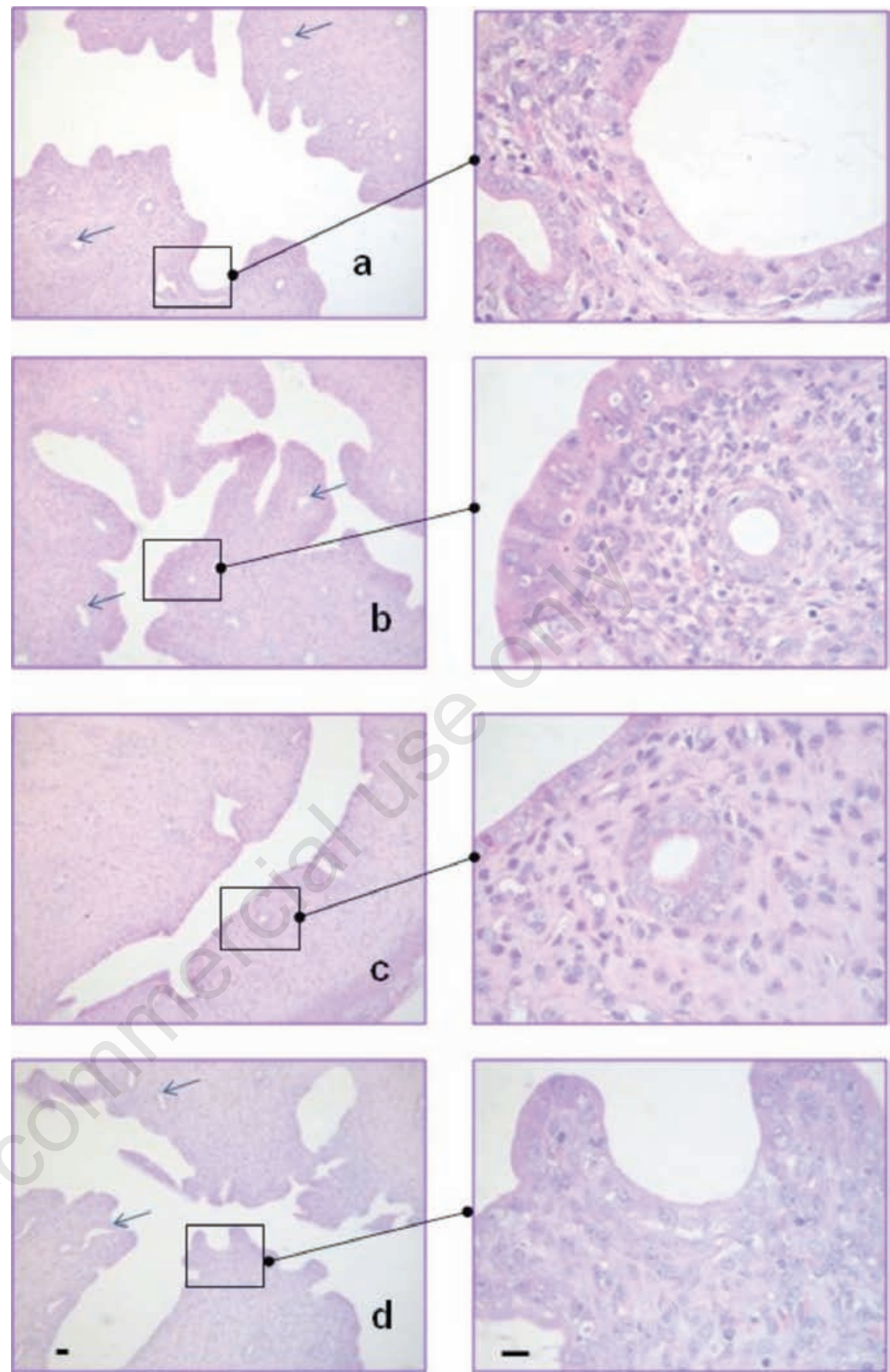

Figure 6. Histamine prevents damage produced by ionising radiation in the rat uterus. A) Normal untreated endometrial wall showing proliferative epithelium, irregular surface and deep endometrial glands (arrows). B) Histamine treated endometrium with similar appearance compared to untreated rats. C) Irradiated rat showing flattened endometrial surface, loss of deep glands and fewer mitotic figures. D) Histamine treated and irradiated rat exhibiting irregular endometrial surface, normal gland number and mitotic figures similar to control animals. Haematoxylin-eosin. 100x and 630x magnification. Scale bar: $20 \mu \mathrm{m}$. 
reported that histamine reduces the degree of bone marrow aplasia caused by ionising radiation that was mediated at least in part by an augment in the rate of proliferation as evidenced by the enhanced PCNA protein expression. ${ }^{11}$ We further determined the effect of histamine and ionising radiation on the intracellular histamine content. Results show that ionising radiation increases intracellular histamine in both untreated and treated animals.

It is important to point out that the radioprotective effect of histamine on the bone marrow and small intestine was demonstrated not only in mouse but also in rats as shown in the present work, ${ }^{10,11}$ suggesting that histamine may produce a cytoprotective action against ionising radiation-induced damage in other mammals.

Despite many years of research there are surprisingly few radiation protectors in use today, whose clinical value is limited due to their toxicity. ${ }^{1}$ Amifostine is the only radioprotective agent that is approved by the Food and Drug Administration (FDA) for preventing gamma irradiation-induced xerostomia in patients under radiotherapy. ${ }^{1,29}$ However, its use encounters two great difficulties: their dose-limiting toxicity and the short period during it is active, which limits amifostine clinical administration. ${ }^{30-32}$ In addition, the clinical use of radioprotectors in radiation therapy continues to be plagued by issues related to possible tumour protection interfering with the irradiation anticancer effect. ${ }^{29,32}$

On the contrary, histamine dihydrochloride (developed as a subcutaneous formulation known as Ceplene) is being used in several clinical trials as an adjuvant with interleukin-2 or interferon alpha therapy for the potential treatment of different types of cancer, including metastatic melanoma, acute myelogenous leukaemia and renal cell carcinoma. In all cases, histamine dihydrochloride was generally well-tolerated and no unexpected or irreversible side effects were reported, demonstrating that histamine dihydrochloride can be safely administered. ${ }^{33,34}$ In addition, this study showed no local or systemic side effects after histamine administration (results not shown).

On the other hand, histamine enhances in vitro radiosensitivity of breast cancer cells through the modulation of antioxidant enzymes. $^{35}$

Therefore, histamine selectively protects ionising radiation-induced cell damage in rat small intestine, bone marrow, salivary glands together with uteri.

The presented evidences indicate that histamine is a potential candidate as a safe radioprotective agent that might increase the therapeutic index of radiotherapy for intra-abdominal and pelvic cancers, and thus enhancing patient quality of life by protecting normal tis-
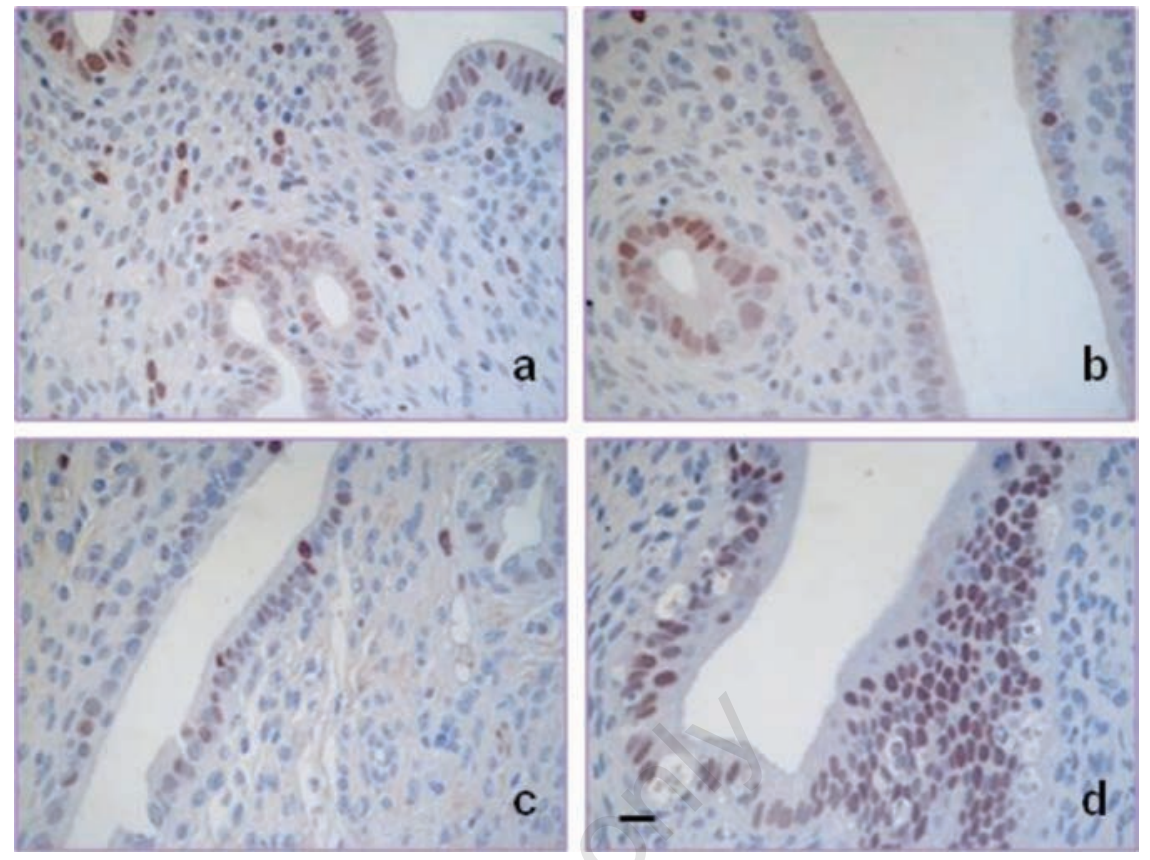

Figure 7. Histamine increases uterine luminal and glandular epithelium proliferation in irradiated animals. Similar PCNA immunoreactivity in uterine luminal and glandular epithelium of A) untreated and B) histamine treated animals. C) Reduced PCNA expression in uterine epithelium of irradiated rats. D) Increased PCNA immunoreactivity in uterine luminal and glandular epithelium of irradiated and histamine treated animals. 630x magnification. Scale bar: $20 \mu \mathrm{m}$.
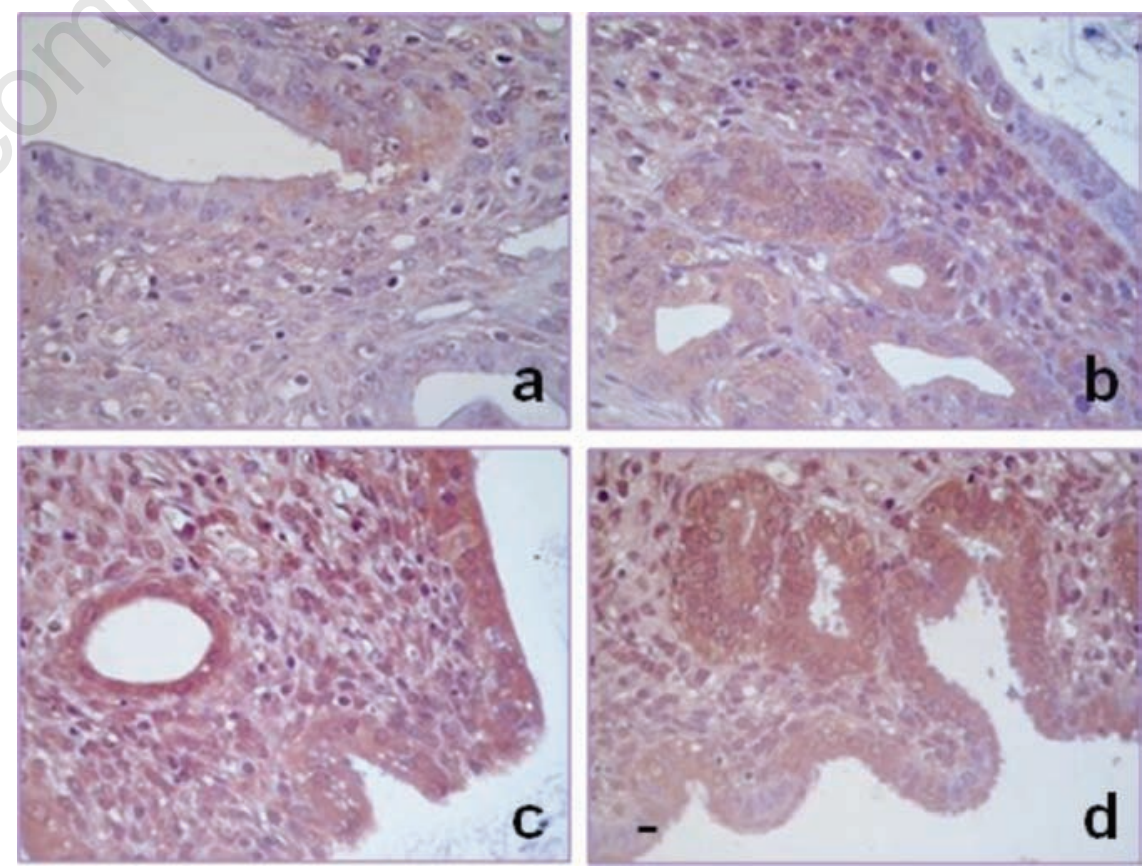

Figure 8. Histamine increases uterine histamine content in irradiated animals. Moderate intracellular content of histamine in rat uteri of A) untreated and B) histamine treated rats. Elevated intracellular histamine content in luminal and glandular cells of C) irradiated and D) irradiated and histamine treated rats. 400x-fold magnification. Scale bar: $20 \mu \mathrm{m}$. 
sue from radiation-induced injury and oxidative DNA damage. However, the efficacy of histamine needs to be carefully investigated in prospective clinical trials.

\section{References}

1. Hall EJ, Giaccia AJ. Radiobiology for the radiologist. 6th ed. Philadelphia: Lippincott Williams \& Wilkins, 2006.

2. Mah LJ, Orlowski C, Ververis K, Vasireddy RS, El-Osta A, Karagiannis TC. Evaluation of the efficacy of radiation-modifying compounds using $\mathrm{YH} 2 \mathrm{AX}$ as a molecular marker of DNA double-strand breaks. Genome integrity $2011 ; 2: 3$.

3. Shirazi A, Ghobadi G, Ghazi-Khansari M. A radiobiological review on melatonin: a novel radioprotector. J Radiat Res 2007;48: 263-72.

4. Wasserman TH, Brizel DM. The role of amifostine as a radioprotector. Oncology 2001;15:349-54.

5. Chawla R, Jaiswal S, Kumar R, Arora R, Sharma RK. Himalayan Bioresource Rhodiola imbricata as a promising radioprotector for nuclear and radiological emergencies. J Pharm Bioallied Sci 2010; 2:213-9.

6. Andreyev HJ, Davidson SE, Gillespie C, Allum WH, Swarbrick E. Practice guidance on the management of acute and chronic gastrointestinal problems arising as a result of treatment for cancer. Gut 2012;61:179-92.

7. Dritschilo A, Sherman DS. Radiation and chemical injury in the bone marrow. Environ Health Perspect 1981;39:59-64.

8. Sudour H, Chastagner P, Claude L, Desandes E, Klein M, Carrie C, et al. Fertility and pregnancy outcome after abdominal irradiation that included or excluded the pelvis in childhood tumor survivors. Int J Radiat Oncol Biol Phys 2010;76:867-73.

9. Wo JY, Viswanathan AN. Impact of radiotherapy on fertility, pregnancy, and neonatal outcomes in female cancer patients. Int J Radiat Oncol Biol Phys 2009;73:1304-12.

10. Medina VA, Croci M, Mohamad N, Massari N, Garbarino G, Cricco P, et al. Mechanisms underlying the radioprotective effect of histamine on small intestine.
Int J Radiat Biol 2007;83:653-63.

11. Medina VA, Croci M, Carabajal E, Bergoc RM, Rivera ES. Histamine protects bone marrow against cellular damage induced by ionising radiation. Int $\mathrm{J}$ Radiat Biol 2010;86:283-90.

12. Medina VA, Prestifilippo JP, Croci M, Carabajal E, Bergoc RM, Elverdin JC, et al. Histamine prevents functional and morphological alterations of submandibular glands exerted by ionising radiation. Int $\mathrm{J}$ Radiat Biol 2011;87:284-92.

13. Medina VA, Rivera ES. Histamine receptors and cancer pharmacology. $\mathrm{Br} \mathrm{J}$ Pharmacol 2010;161:755-67.

14. Parsons ME, Ganellin CR. Histamine and its receptors. Br J Pharmacol 2006;1:12735.

15. Sander LE, Lorentz A, Sellge G, Coëffier M, Neipp M, Veres T, et al. Selective expression of histamine receptors H1R, H2R, and H4R, but not H3R, in the human intestinal tract. Gut 2006;55:498-504.

16. Maintz L, Schwarzer V, Bieber T, van der Ven K, Novak N. Effects of histamine and diamine oxidase activities on pregnancy: a critical review. Hum Reprod Update 2008; 5:485-95.

17. Pos Z, Hegyesi H, Rivera E. Histamine and cell proliferation, pp.199-217. In: A. Falus (ed.) Histamine: Biology and Medical Aspects. 1st ed. Budapest: SpringMed Publishing Ltd., 2004.

18. Pinto MM, Santos NF, Amaral A. Current status of biodosimetry based on standard cytogenetic methods. Radiat Environ Biophys 2010;49:567-81.

19. Kasai H. Analysis of a form of oxidative DNA damage, 8-hydroxy-2'-deoxyguanosine, as a marker of cellular oxidative stress during carcinogenesis. Mutat Res 1997;387:147-63.

20. Vanhauwaert A, Vanparys P, Kirsch-Volders M. The in vivo gut micronucleus test detects clastogens and aneugens given by gavage. Mutagenesis 2001;16:39-50.

21. Fenech $M$. The in vitro micronucleus technique. Mutat Res 2000;455:81-95.

22. Potten CS, Wilson JW, Booth C. Regulation and significance of apoptosis in the stem cells of the gastrointestinal epithelium. Stem Cells 1997;15:82-93.

23. Potten CS, Owen G, Booth D. Intestinal stem cells protect their genome by selective segregation of template DNA strands.
J Cell Sci 2002;115:2381-8.

24. Potten CS. Stem cells in gastrointestinal epithelium: numbers, characteristics and death. Philos Trans R Soc Lond B Biol Sci 1998;353:821-30.

25. Fujimoto K, Imamura I, Granger DN, Wada H, Sakata T, Tso P. Histamine and histidine decarboxylase are correlated with mucosal repair in rat small intestine after ischemia-reperfusion. J Clin Invest 1992; 89:126-33.

26. Yoshida T, Iwakiri R, Noda T, Okamoto K, Kojima M, Fukuyama K, et al. Histaminergic effect on apoptosis of rat small intestinal mucosa after ischemia-reperfusion. Dig Dis Sci 2000:45;1138-44.

27. Prasad KN. Radiation syndromes and their modifications, pp. 123-152. In: KN Prasad (ed.) Handbook of radiobiology. 2nd ed. CRC Press, Inc., 1995.

28. Critchley HO, Wallace WH. Impact of cancer treatment on uterine function. J Natl Cancer Inst 2005;34:64-8.

29. Grdina DJ, Murley JS, Kataoka Y, Baker KL, Kunnavakkam R, Coleman MC, et al. Amifostine induces antioxidant enzymatic activities in normal tissues and a transplantable tumor that can affect radiation response. Int J Radiat Oncol Biol Phys 2009;73:886-96.

30. Dziegielewski J, Baulch JE, Goetz W, Coleman MC, Spitz DR, Murley JS, et al. WR-1065, the active metabolite of amifostine, mitigates radiation-induced delayed genomic instability. Free Rad Biol Med 2008;45:1674-81.

31. Grdina DJ, Murley JS, Kataoka Y. Radioprotectans: current status and new directions. Oncology 2002;63:2-10.

32. Weiss JF, Landauer MR. History and development of radiation-protective agents. Int J Radiat Biol 2009:85;539-73.

33. Galmarini CM. Histamine dihydrochloride (subcutaneous) Maxim. Curr Opin Investig Drugs 2004;12:1298-310.

34. Yang LP, Perry CM. Histamine dihydrochloride: in the management of acute myeloid leukaemia. Drugs 2011;71:109-22.

35. Medina VA, Cricco G, Nuñez M, Martín G, Mohamad N, Correa-Fiz F, et al. Histamine-mediated signaling processes in human malignant mammary cells. Cancer Biol Ther 2006;5,1462-71. 